\title{
Methods for the calculation of thermoacoustic stability margins and Monte Carlo-free uncertainty quantification
}

\author{
Georg A. Mensah* \\ Institut fr Strmungsmechanik \\ und Technische Akustik \\ Technische Universiterlin \\ Berlin, Germany \\ Email: georg.a.mensah@tu-berlin.de
}

\author{
Luca Magri \\ University of Cambridge \\ Engineering Department \\ Cambridge, UK
}

\author{
Jonas P. Moeck \\ Institut fr Strmungsmechanik \\ und Technische Akustik \\ Technische Universiterlin \\ Berlin, Germany
}

\begin{abstract}
Thermoacoustic instabilities are a major threat for modern gas turbines. Frequency-domain based stability methods, such as network models and Helmholtz solvers, are common design tools because they are fast compared to compressible CFD computations. Frequency-domain approaches result in an eigenvalue problem, which is nonlinear with respect to the eigenvalue. Nonlinear functions of the frequency are, for example, the $n-\tau$ model, impedance boundary conditions, etc. Thus, the influence of the relevant parameters on mode stability is only given implicitly. Small changes in some model parameters, which are obtained by experiments with some uncertainty, may have a great impact on stability. The assessment of how parameter uncertainties propagate to system stability is therefore crucial for safe gas turbine operation. This question is addressed by uncertainty quantification. A common strategy for uncertainty quantification in thermoacoustics is risk factor analysis. It quantifies the uncertainty of a set of parameters in terms of the probability of a mode to become unstable.

One general challenge regarding uncertainty quantification is the sheer number of uncertain parameter combinations to be quantified. For instance, uncertain parameters in an annular combustor might be the equivalence ratio, convection times, geometrical parameters, boundary impedances, flame response model parameters etc. Assessing also the influence of all possible combinations of these parameters on the risk factor is a numerically very costly task.

A new and fast way to obtain algebraic parameter models in order to tackle the implicit nature of the eigenfrequency problem is using adjoint perturbation theory. Though adjoint perturbation methods were recently applied to accelerate the risk factor analysis, its potential to improve the theory has not yet been fully exploited. This paper aims to further utilize adjoint methods for the quantification of uncertainties. This analytical method avoids the usual random Monte Carlo simulations, making it particularly attractive for industrial purposes. Using network models and the open-source Helmholtz solver PyHoltz it is also discussed how to apply the method with standard modeling techniques. The theory is exemplified based on a simple ducted flame and a combustor of EM2C laboratory for which experimental validation is available.
\end{abstract}

${ }^{*}$ Address all correspondence to this author. 


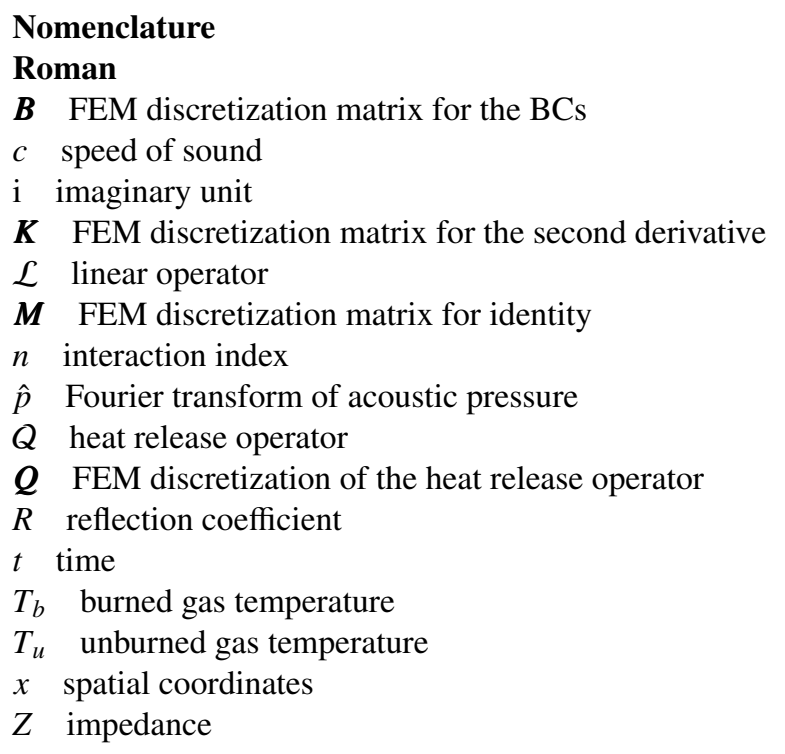

\section{Greek}

$\gamma$ ratio of specific heats

$\varepsilon$ perturbation parameter

$\boldsymbol{\varepsilon}$ parameter vector

$\rho$ fluid density

$\tau$ time delay

$\omega$ complex eigenfrequency

Abbreviations

BC Boundary Condition

FEM Finite Element Method

FTF Flame Transfer Function

MC Monte Carlo

PDF Probability Density Function

RF Risk Factor

The primary text heading is boldface and flushed left with the left margin. The spacing between the text and the heading is two line spaces.

\section{INTRODUCTION}

Thermoacoustic instabilities are a major threat for modern gas turbines [1]. One approach to assess thermoacoustic stability is the use of Large Eddy Simulation (LES) [2,3]. However, this approach is too numerically expensive to be used as a design tool for parametric studies. Therefore, faster frequency-domain based methods such as low-order network models $[4,5,6,7]$ and thermoacoustic Helmholtz solvers [8,9] are utilized in academia and industry. Recently, high-frequency instabilities were successfully tackled with frequency-domain linearized Euler equation solvers [10]. Frequency-domain based methods result in an eigenvalue problem that is nonlinear with respect to the eigenvalue. This can be represented in compact form as $[11,12]$

$$
\mathcal{L}(\omega, \boldsymbol{\varepsilon}) \widehat{p}(\boldsymbol{x})=0
$$

Here, $\mathcal{L}$ denotes the Fourier-transformed operator associated with the linearized time-domain problem, $\omega$ denotes the complex eigenfrequency, which appears in a nonlinear fashion, $\boldsymbol{\varepsilon}$ is a vector containing the model parameters, and $\widehat{p}(\boldsymbol{x})$ is the Fourier transform of the acoustic pressure, following the convention $p(\boldsymbol{x}, t)=\widehat{p}(\boldsymbol{x}) \exp (\mathrm{i} \omega t)$. With this convention, if the imaginary part of the eigenvalue, $\omega_{i}$, is negative, the system is linearly unstable. The angular frequency is provided by the real part, $\omega_{r}$. (The spatial dependency is dropped for brevity from now on, i.e., $\widehat{p}(\boldsymbol{x})=\widehat{p}$.) By definition, the eigenfrequencies are such that $\mathcal{L}(\omega, \boldsymbol{\varepsilon})$ has a non-empty kernel. In the finite-dimensional case, which arises when the operator is discretized or 
when a network model is considered, this corresponds to the requirement $\operatorname{det} \mathcal{L}=0$. This defines an implicit relation between the eigenvalues and the parameters:

$$
F(\omega, \boldsymbol{\varepsilon})=0 .
$$

The thermoacoustic stability problem, in line with other engineering and physics stability problems, can be stated as: Given a set of parameters for the combustor geometry, flame response and acoustic properties, will the system exhibit thermoacoustic oscillations? Mathematically, this means to determine whether the eigenvalue lies in the stable or in the unstable half plane, which requires solving for the dispersion relation (2) and evaluate whether there are unstable eigenfrequencies. There are different numerical methods for solving the dispersion relation or the eigenproblem (1), some of which are described in [8].

The first objective of this paper is to propose an analytical method for the calculation of the stability margin, which provides the different combinations of parameters for which the thermoacoustic system is marginally stable, i.e.,

$$
F\left(\omega_{i}=0, \boldsymbol{\varepsilon}_{\text {mar }}\right)=0 .
$$

The feature that makes thermoacoustic stability calculation particularly challenging is the high sensitivity of the eigenvalues to the parameters of the system. For example, small changes or uncertainty on the equivalence ratio, convection times, geometrical parameters, boundary impedances, flame response model parameters $[13,14,15]$ can significantly affect the eigenfrequencies, which might undermine the reliability of the predictions. Therefore, having methods to calculate this sensitivity information is as important as having methods for the stability calculation.

The second objective of this paper is to present a method for the accurate and fast calculation of such sensitivities, which is based on adjoint-based perturbation theory $[13,12,11]$.

Quantification of the uncertainties in all parameters with respect to the stability is therefore identified as one of the key challenges in the field of thermoacoustic stability analysis [16]. A common strategy to evaluate how the parameter uncertainties affect the growth rate is risk factor analysis $[17,18]$, which is performed by classical Monte Carlo methods. Monte Carlo methods are based on random samples of the (eigen)problem and can be expensive when the required number of samples is high. The risk factor quantifies the probability that a thermoacoustic mode is unstable given an uncertainty in the system parameters.

One general challenge regarding uncertainty quantification is posed by the sheer number of uncertain parameter combinations to be quantified. Therefore, assessing also the influence of all possible combinations of these parameters on the risk factor is a numerically costly task. This so-called "curse of dimensionality" can be tackled by calculating explicit algebraic models (surrogate models) for the growth rate, for example, based on nonlinear regression of a small Monte Carlo sample, which circumvents the implicit nature of the eigenvalue problem. Finally, the risk factor can be calculated by a Monte Carlo experiment on the simple algebraic model $[18,17,19]$.

By observing that the uncertainty quantification problem is akin, but not equal, to sensitivity analysis from a probabilistic standpoint, more accurate and faster methods were proposed. Indeed, another way to obtain explicit algebraic models for the eigenfrequency can be devised by using spectral perturbation theory. Originally developed in the field of quantum mechanics to solve eigenvalue problems associated with the Schrdinger equation [20], spectral perturbation theory has become a powerful tool in a variety of science and engineering problems. In hydrodynamic stability the review by [21] provides an excellent overview of the many applications of these theories. Adjoint-based methods were developed for thermoacoustic sensitivity by Magri and Juniper [13]. Recently, it was applied to accelerate the uncertainty quantification calculation by using the gradient information extracted from the adjoint operator [14,15]. Multi-parameter perturbation expansions were also recently used to optimize the damper distribution of a generic annular combustion chamber [11]. The use of more than one perturbation parameter appears to be valuable for uncertainty quantification purposes, too. The third objective of this paper is to exploit multi-parameter adjoint-based methods further for the calculation of the risk factor by avoiding Monte Carlo simulation altogether.

First the theoretical fundamentals of the theory are introduced and discussed. It is then applied to Rijke tube and laboratory scale combustor. The paper concludes with remarks on how the theory may be further developed.

\section{ADJOINT PERTURBATION THEORY}

First, the theory is presented for the case of a system with a single parameter. Afterwards, the theory is generalized to the case of a system with multiple parameters, which is particularly relevant to thermoacoustics. 


\subsection{Systems with one parameter}

Adjoint perturbation theory addresses the question of how the eigenfrequency $\omega$ and the eigenmode $\widehat{p}$ change when a parameter $\varepsilon$ is changed by a perturbation $\Delta \varepsilon$, under the constraint of Eq. (1). Based on the known solution of the unperturbed problem $\mathcal{L}\left(\omega_{0}, \varepsilon_{0}\right) \widehat{p}_{0}=0$, asymptotic power series for the eigenfrequency $\omega$ and the corresponding pressure mode $\widehat{p}$ are assumed to model their respective change due to a change $\Delta \varepsilon$ in a model parameter $\varepsilon$

$$
\begin{gathered}
\omega=\omega_{0}+\sum_{n=1}^{N} \omega_{n}(\Delta \varepsilon)^{n}, \\
\widehat{p}=\widehat{p}_{0}+\sum_{n=1}^{N} \widehat{p}_{n}(\Delta \varepsilon)^{n},
\end{gathered}
$$

where $N$ is the order at which the series is truncated. The series expansions are approximations to the solutions of the perturbed eigenvalue problem

$$
\mathcal{L}\left(\omega, \varepsilon_{0}+\Delta \varepsilon\right) \widehat{p}=0 .
$$

While $\omega_{0}$ and $\widehat{p}_{0}$ are the solutions of the unperturbed problem, the coefficients $\omega_{n}$ and $\widehat{p}_{n}$ can be obtained after (i) expanding the perturbed eigenvalue problem (6) into a Taylor series centered at $\omega_{0}$ and $\varepsilon_{0}$, (ii) substituting the power series (4) and (5) into this expansion and (iii) grouping powers of $\Delta \varepsilon$. For each order $n$ this procedure yields an equation of the form

$$
\mathcal{L}_{0,0} \widehat{p}_{n}=-r_{n}-\omega_{n} \mathcal{L}_{1,0} \widehat{p}_{0} .
$$

Here, $\mathcal{L}_{k, l}$ is a short hand notation for $\frac{1}{k !} \frac{1}{l !} \frac{\partial^{k+l}}{\partial \omega^{k} \partial \varepsilon} \mathcal{L}\left(\omega_{0}, \varepsilon_{0}\right)$ and $r_{n}$ abbreviates a term consisting of coefficients $\omega_{j}$ and $\widehat{p}_{j}$ of order $j<n$ only. For instance, for first and second orders the following $r_{n}$ are found:

$$
\begin{aligned}
& r_{1}=\mathcal{L}_{0,1} \widehat{p}_{0}, \\
& r_{2}=\mathcal{L}_{0,1} \widehat{p}_{1}+\mathcal{L}_{0,2} \widehat{p}_{0}+\omega_{1}\left(\mathcal{L}_{1,0} \widehat{p}_{1}+\mathcal{L}_{1,1} \widehat{p}_{0}\right)+\omega_{1}^{2} \mathcal{L}_{2,0} \widehat{p}_{0} .
\end{aligned}
$$

With an incremental scheme Eq. (7) can be solved for $\omega_{n}$ and $\widehat{p}_{n}$. First, the adjoint eigenvector $\widehat{p}_{0}^{\dagger}$ corresponding to $\widehat{p}_{0}$ has to be computed. This is obtained from the solution of the adjoint problem

$$
\mathcal{L}^{\dagger}\left(\omega_{0}, \varepsilon_{0}\right) \widehat{p}_{0}^{\dagger}=0
$$

where the operator $\mathcal{L}^{\dagger}$ is the adjoint operator of $\mathcal{L}$, i.e., the operator satisfying $\langle\mathcal{L} x \mid y\rangle=\left\langle x \mid \mathcal{L}^{\dagger} y\right\rangle$. Here $x$ and $y$ are arbitrary elements from the respective function space and $\langle\cdot \mid \cdot\rangle$ denotes an inner product of this space. In the current context, the inner product is an integral over the domain $\Omega$ on which the thermoacoustic Helmholtz equation is considered: $\langle x \mid y\rangle=\int_{\Omega} x y^{*} \mathrm{~d} \Omega$. In a discretized setting, the corresponding function space is finite dimensional, and the adjoint operator then simply corresponds to the Hermitian transpose of the direct operator, meaning that the adjoint operator is simply the complex transpose of the direct operator, $\mathcal{L}^{\dagger}=\mathcal{L}^{* T}$.

The corrections to the unperturbed eigenfrequency, $\omega_{n}$, are obtained by taking then the inner product of Eq. (7) with the adjoint eigenvector

$$
\left\langle\widehat{p}_{0}^{\dagger} \mid r_{n}\right\rangle+\omega_{n}\left\langle\widehat{p}_{0}^{\dagger} \mid \mathcal{L}_{1,0}\right\rangle=0
$$

These corrections are then substituted back into Eq. (7) to obtain the correction for the eigenvectors, $\widehat{p}_{n}$. If the considered mode is non-degenerate this results in the following expressions for the eigenvalue corrections:

$$
\omega_{n}=-\frac{\left\langle\widehat{p}_{0}^{\dagger} \mid \mathcal{L}_{1,0}\right\rangle}{\left\langle\widehat{p}_{0}^{\dagger} \mid r_{n}\right\rangle} .
$$




\subsection{Systems with multiple parameters}

Uncertainty quantification deals with multiple parameters that are varied simultaneously. The theory presented in the previous section can be extended to multi-parameter problems with a simple trick. Consider $P$ different parameters $\varepsilon_{1}, \ldots, \varepsilon_{P}$. Let therefore $\varepsilon=\left[\begin{array}{lll}\varepsilon_{1} & \ldots & \varepsilon_{P}\end{array}\right]^{\mathrm{T}}$ denote a parameter vector. At first order the eigenvalue drift may then be compared with the first-order Taylor series

$$
\omega \approx \omega_{0}+\sum_{p=1}^{P} \omega_{1, p} \varepsilon_{p}=\omega_{0}+\left.\frac{\partial \omega}{\partial \boldsymbol{\varepsilon}}\right|_{\varepsilon=\varepsilon_{0}} \Delta \boldsymbol{\varepsilon}
$$

This comparison reveals that the coefficients $\omega_{1, p}$ are equal to the partial derivatives of $\omega$ at $\boldsymbol{\varepsilon}_{0}$. Thus, the derivatives can be computed from single-parameter series and then used in a multi-parameter study. However, this rationale only works if the eigenvalue is totally differentiable with respect to the considered parameters, a condition that does not hold true for degenerate cases, such as in rotationally symmetric annular combustors. These, however are not considered in this present paper and the extension is left for future work.

For non-degenerate eigenvalues, such as the systems considered here, second-order formulae can be derived similar to the single-parameter case, by establishing multivariate quadratic polynomial ansätze for the eigenfrequency

$$
\begin{gathered}
\omega=\omega_{0}+\sum_{i}^{P} \omega_{1: i} \Delta \varepsilon_{i}+\sum_{i=1}^{P} \sum_{j=1}^{i} \omega_{2: i j} \Delta \varepsilon_{i} \Delta \varepsilon_{j}, \\
\widehat{p}=\widehat{p}_{0}+\sum_{i}^{P} \widehat{p}_{1: i} \Delta \varepsilon_{i}+\sum_{i=1}^{P} \sum_{j=1}^{i} \widehat{p}_{2: i j} \Delta \varepsilon_{i} \Delta \varepsilon_{j},
\end{gathered}
$$

and expanding the eigenvalue problem into a second-order Taylor series:

$$
\begin{aligned}
\mathcal{L}(\omega, \boldsymbol{\varepsilon}) \approx & \mathcal{L}\left(\omega_{0}, \boldsymbol{\varepsilon}_{0}\right)+\underbrace{\frac{\partial}{\partial \omega} \mathcal{L}\left(\omega_{0}, \boldsymbol{\varepsilon}_{0}\right)}_{:=\mathcal{L}_{1,0}}\left(\omega-\omega_{0}\right)+\underbrace{\frac{\partial^{2}}{\partial \omega^{2}} \mathcal{L}\left(\omega_{0}, \boldsymbol{\varepsilon}_{0}\right)}_{:=\mathcal{L}_{2,0}}\left(\omega-\omega_{0}\right)^{2} \\
& +\sum_{i=1}^{P} \underbrace{\frac{\partial}{\partial \varepsilon_{i}} \mathcal{L}\left(\omega_{0}, \boldsymbol{\varepsilon}_{0}\right)}_{:=\mathcal{L}_{0,1: i}} \Delta \varepsilon_{i}+\sum_{i=1}^{P} \underbrace{\frac{\partial^{2}}{\partial \omega \partial \varepsilon_{i}} \mathcal{L}\left(\omega_{0}, \boldsymbol{\varepsilon}_{0}\right)}_{:=\mathcal{L}_{1,1: i}}\left(\omega-\omega_{0}\right) \Delta \varepsilon_{i} \\
& +\sum_{i=1}^{P} \sum_{j=1}^{i} \underbrace{\frac{\partial^{2}}{\partial \varepsilon_{i} \partial \varepsilon_{j}} \mathcal{L}\left(\omega_{0}, \boldsymbol{\varepsilon}_{0}\right)}_{:=\mathcal{L}_{0,2: i j}} \Delta \varepsilon_{i} \Delta \varepsilon_{j} .
\end{aligned}
$$

Again, substitution of the ansätze into the eigenvalue expansion yields at first order

$$
\mathcal{L}_{0,0} \widehat{p}_{1: i}=-\mathcal{L}_{0,1: i} \widehat{p}_{0}-\omega_{1: i} \mathcal{L}_{1,0} \widehat{p}_{0}
$$

and for the second order

$$
\mathcal{L}_{0,0} \widehat{p}_{2: i j}=-r_{2: i j}-\omega_{2: i j} \mathcal{L}_{1,0} \widehat{p}_{0},
$$


where

$$
r_{2: i j}=\left\{\begin{array}{l}
\text { if } i=j: \\
\mathcal{L}_{0,1: i} \widehat{p}_{1: i}+\mathcal{L}_{0,2: i i} \widehat{p}_{0}+\omega_{i}\left(\mathcal{L}_{1,0} \widehat{p}_{1: i}+\mathcal{L}_{1,1: i} \widehat{p}_{0}\right)+\omega_{1: i}^{2} \mathcal{L}_{2,0} \widehat{p}_{0} \\
\text { if } i \neq j: \\
\mathcal{L}_{0,1: i} \widehat{p}_{1: j}+\mathcal{L}_{0,1: j} \widehat{p}_{1: i}+\mathcal{L}_{0,2: i j} \widehat{p}_{0}+\mathcal{L}_{1,0}\left(\widehat{p}_{1: i} \omega_{1: j}+\widehat{p}_{1: j} \omega_{1: i}\right) \\
+\omega_{1: i} \mathcal{L}_{1,1: j} \widehat{p}_{0}+\omega_{1: j} \mathcal{L}_{1,1: i} \widehat{p}_{0}+2 \omega_{1: i} \omega_{1: j} \mathcal{L}_{2,0} \widehat{p}_{0} .
\end{array}\right.
$$

Equivalent to the single-parameter case, the first-order Eqns. (17) are first solved for $\omega_{1: i}$ and then $\widehat{p}_{1: i}$ by taking the inner product with the adjoint eigenmode. Subsequently, the second-order Eqns. (18) can be solved for $\omega_{2: i j}$ and $\widehat{p}_{2: i j}$, by applying the same operation.

\section{FORWARD UNCERTAINTY QUANTIFICATION}

Sources of significant uncertainties in thermoacoustic models are the flame transfer function and the acoustic damping. Here, we calculate how the thermoacoustic growth rate, $-\operatorname{Im}(\omega)$, which governs the stability, is affected by uncertain flame parameters. Studying uncertainty quantification for the acoustic damping, for example through impedance boundary conditions, is just as straightforward [15]. This is called "forward problem", as opposed to the "inverse problem" [22], because the focus is on how uncertainties in the input parameters affect the output, which is the eigenfrequency. We assume we know the maximum and minimum values of the uncertain flame parameters. Using the Principle of Maximum Entropy, we choose the uniform distribution for the input parameters because it is the least biased possible distribution given the available information [23]. However, the methods proposed do not rely on the presumed PDF; therefore other PDFs can be used without adaptation of the proposed techniques. Interestingly, Ndiaye et al. [17] have shown that the PDF shape has a minor effect on the risk factor in the case they considered.

The outputs of forward uncertainty quantification in this study is the risk factor

$$
\mathrm{RF}=\int_{-\infty}^{0} \operatorname{PDF}\left(\omega_{i}\right) \mathrm{d} \omega_{i}
$$

which is the probability that the system is unstable given a (mutli-variate) PDF $f$ of the flame parameters

$$
P(\boldsymbol{\varepsilon} \in E)=\int_{E} f(\boldsymbol{\varepsilon}) \mathrm{d} \boldsymbol{\varepsilon},
$$

where $P$ is the probability for the parameters $\boldsymbol{\varepsilon}$ to take values in $E$. In forward uncertainty quantification, Eq. (20) is the output of the analysis and Eq. (21) is the input.

A common way to perform uncertainty quantification on the basis of a thermoacoustic model is the standard Monte Carlo method [19,17]. With this method, $M$ random values of $\boldsymbol{\varepsilon}$ are selected according to their PDFs, and the full nonlinear eigenproblem (1) is solved $M$ times to provide $M$ eigenvalues. This means that with this method, $M$ nonlinear eigenproblems have to be solved, with $M \sim O\left(10^{4}-10^{5}\right)$, which is a time-consuming task. The Monte Carlo method always converges to the final PDF but suffers from slow $O(1 / \sqrt{M})$-convergence, which could be prohibitive for large systems such as Helmholtz or linearized Euler models in complex geometries.

In view of this, Monte Carlo-free methods are presented to calculate the uncertainty of the thermoacoustic stability margin of the combustor. These methods consist of two steps:

1. Calculation of the stability margin, i.e., the flame parameters for which $F\left(\omega_{i}=0, \boldsymbol{\varepsilon}\right)=0$ starting from the unperturbed condition;

2. Analytical integration of the unstable portion defined by the intersection of the stability margin and the domain of the assumed PDF of the flame parameters. 
As an example, in a two-parameter model with a uniform distribution of the parameters, the PDF reads

$$
f\left(\varepsilon_{1}, \varepsilon_{2}\right)=\left\{\begin{array}{l}
\text { if } \boldsymbol{\varepsilon} \in\left[\varepsilon_{1 \min }, \varepsilon_{1 \max }\right] \times\left[\varepsilon_{2 \min }, \varepsilon_{2 \max }\right]: \\
\frac{1}{\left(\varepsilon_{1 \max }-\varepsilon_{1 \min }\right)\left(\varepsilon_{2 \max }-\varepsilon_{2 \min }\right)} \\
\text { else : } \\
0
\end{array}\right.
$$

for the probability density function of the parameters and

$$
\omega\left(\varepsilon_{1}, \varepsilon_{2}\right)=c_{0}+c_{1} \varepsilon_{1}+c_{2} \varepsilon_{2}
$$

as an eigenfrequency model. The unstable parameter region $E_{\mathrm{unst}}$ is defined by the parameters for which $\omega_{i}<0$. The integral in Eq. (27) to compute the risk factor hence amounts to a simple area evaluation. If, for instance, the set of admissible parameters $\varepsilon_{1}$ and $\varepsilon_{2}$ is divided into two different areas, the ratio of the area lying in the unstable area and the total admissible area is the risk factor. Figure 1 schematically illustrates this case in a two-parameter space.

Two methods are proposed in the subsequent sections to determine the stability margin.

\subsection{Stability margin calculation}

\subsubsection{Method 1: Nonlinear-eigenproblem-based method}

This method serves as a benchmark solution and it is not based on adjoint theory, i.e., no gradients are calculated. First, a small uniform grid of flame parameters centered at the unperturbed condition is assumed. Second, the full nonlinear eigenproblem in Eq. (1) is solved for each set of flame parameters. Third, the locus of parameters for which $\omega_{i}=0$ is identified by curve fitting. Note that, although this method needs the solution of several nonlinear eigenproblems, it is computationally less demanding than a Monte Carlo method because the number of grid points used is much smaller than the random samples required in the Monte Carlo method.

\subsubsection{Method 2: Adjoint-based method}

An algebraic model for the eigenfrequency (23) is derived by means of adjoint perturbation theory as outlined in the previous section. The coefficients for the algebraic model of the eigenfrequency in Eq. (23) are obtained by using the adjoint eigenvectors, as follows

$$
c_{i}=\frac{\partial \omega}{\partial \varepsilon_{i}}=-\frac{\left\langle\widehat{p}_{0}^{\dagger} \mid \partial_{\omega} \mathcal{L} \widehat{p}_{0}\right\rangle}{\left\langle\widehat{p}_{0}^{\dagger} \mid \partial_{\varepsilon_{i}} \mathcal{L} \widehat{p}_{0}\right\rangle}
$$

If a second order model is used, we have

$$
\omega=\omega_{0}+c_{1} \varepsilon_{1}+c_{2} \varepsilon_{2}+c_{11} \varepsilon_{1}^{2}+c_{12} \varepsilon_{1} \varepsilon_{2}+c_{22} \varepsilon_{2}^{2}
$$

The coefficients can be computed by second order adjoint multi-parameter theory according to the equations 17 and 18 . Because of the second order the stability margin is then a conic section in the parameter space.

\subsection{Evaluation of the risk factor in the parameter space}

In previous studies, the risk factor has been computed by means of Monte Carlo simulations [19, 17, 14, 15]. Although adjoint-based and algebraic models were derived, the risk factor was obtained by Monte Carlo simulations. In this section, a Monte Carlo-free method is proposed.

If there is an explicit algebraic expression $\omega=\omega(\boldsymbol{\varepsilon})$ available, modeling the dependence of the frequency of interest from model parameters, the set of unstable parameter configurations can be defined as follows

$$
E_{\text {unst. }}=\left\{\boldsymbol{\varepsilon} \in \mathbb{R}^{P} \mid \operatorname{Im}(\omega(\boldsymbol{\varepsilon}))<0\right\},
$$


where $P$ is the number of parameters. Consequently, with this definition the risk factor can be computed as

$$
\mathrm{RF}=\int_{E_{\text {unst. }}} f(\boldsymbol{\varepsilon}) \mathrm{d} \boldsymbol{\varepsilon}
$$

Note that this method enables the calculation of the risk factor directly in the parameter space, as opposed to the standard Monte Carlo methods of [19] and the adjoint-based methods of [12]. This integral yields a closed expression for the risk factor computation with no Monte Carlo sampling. In summary, the risk factor computation can therefore be based on a single solution of the eigenvalue problem following these steps:

1. Solve the nonlinear eigenvalue problem (1);

2. Solve the corresponding adjoint eigenvalue problem (10);

3. Compute the eigenvalue sensitivities by means of adjoint perturbation theory for the algebraic model of the eigenfrequency.

4. Evaluate the risk factor in Eq. (21).

\section{THERMOACOUSTIC MODELS}

The governing equations are the continuity, momentum, and the energy equations, which, by neglecting viscosity, heat conduction and advection, read

$$
\begin{gathered}
\frac{\partial \rho}{\partial t}+u \frac{\partial \rho}{\partial x}+\rho \frac{\partial u}{\partial x}=0 \\
\rho \frac{\partial u}{\partial t}+\rho u \frac{\partial u}{\partial x}+\frac{\partial p}{\partial x}=0 \\
\frac{\partial p}{\partial t}+u \frac{\partial p}{\partial x}+\gamma p \frac{\partial u}{\partial x}=0
\end{gathered}
$$

Based on the linearized version of these equations, two different low-order models are utilized.

\subsection{Wave-based approach}

The model assumes homogeneous properties along each segment, hence the flame or changes in area split the domain into segments connected by jump conditions in a small Helmholtz number limit (the area variations/flame length being compact with respect to the acoustic wavelength). These relations are derived from Eq. (28) assuming a steady state and integrating from one side of the discontinuity to the other

$$
\begin{aligned}
& \llbracket \rho u \rrbracket=0, \\
& \llbracket p+\rho u^{2} \rrbracket=0, \\
& \llbracket \frac{\gamma}{(\gamma-1)} p u+\frac{1}{2} \rho u^{3} \rrbracket=q A,
\end{aligned}
$$

where $q$ is the heat release per unit area, and $A$ is the area of the section before the flame. Linearizing the governing equations around the mean flow and assuming a zero Mach number limit (i.e $\bar{u}=0)$ provides the steady base flow equations and the time-varying fluctuation equations. The mean-flow pressure is constant and uniform across the discontinuities. Furthermore, from the energy equation it ensures that the steady heat release is zero, $\bar{q}=0$. Therefore, to model the mean flow temperature jump, an artificial increment in temperature $(\Delta \bar{T})$ is imposed. The linearized equations read

$$
\begin{gathered}
\bar{\rho} \frac{\partial u^{\prime}}{\partial t}+\frac{\partial p^{\prime}}{\partial x}=0 \\
\frac{\partial p^{\prime}}{\partial t}+\gamma \bar{p} \frac{\partial u^{\prime}}{\partial x}=0
\end{gathered}
$$


with the following jump conditions

$$
\begin{aligned}
& \llbracket p^{\prime} \rrbracket=0, \\
& \llbracket u^{\prime} \rrbracket=A \frac{\gamma-1}{\gamma \bar{p}} \int_{b^{-}}^{b^{+}} q^{\prime} \mathrm{d} x .
\end{aligned}
$$

The unsteady heat release is modeled by an $n-\tau$ model proportional to the velocity upstream of the flame [24]

$$
q^{\prime}(x, t)=\beta u^{\prime}\left(b^{-}, t-\tau\right) \delta(x-b),
$$

where $\beta$ is the flame strength, $\tau$ the time delay and $\delta(x-b)$ is the Dirac delta. The flame strength $\beta$ can be expressed as the dimensionless interaction index $n$ and the ratio of mean heat release $q_{\text {total }}$ and bulk velocity $U_{\text {bulk }}$ as

$$
\begin{aligned}
& q^{\prime}=\underbrace{\frac{q_{\text {total }}}{U_{\text {bulk }}} n u^{\prime}\left(b^{-}, t-\tau\right) \delta(x-b),}_{=\beta} \\
& \frac{q_{\text {total }}}{U_{\text {bulk }}}=p_{0}\left(\frac{T_{\mathrm{b}}}{T_{\mathrm{u}}}-1\right) \frac{\gamma}{\gamma-1} A .
\end{aligned}
$$

Using the wave-based approach, the acoustic variables are expressed in each segment $i$ as waves

$$
\begin{aligned}
& p_{i}^{\prime}(x, t)=A_{i}^{+}\left(t-\frac{x}{\bar{c}_{i}}\right)+A_{i}^{-}\left(t+\frac{x}{\bar{c}_{i}}\right), \\
& u_{i}^{\prime}(x, t)=\frac{1}{\rho_{i} \bar{c}_{i}}\left[A_{i}^{+}\left(t-\frac{x}{\bar{c}_{i}}\right)-A_{i}^{-}\left(t+\frac{x}{\bar{c}_{i}}\right)\right],
\end{aligned}
$$

where $A_{i}^{+}$is the downstream propagating acoustic wave, while $A_{i}^{-}$propagates upstream $(\mathrm{x}=0)$. By substituting the boundary conditions, which relate the downstream wave exiting the domain to the reflected wave, into the linearized jump conditions (33) and Fourier-transforming the problem, yields a nonlinear eigenproblem in $\omega, \mathcal{L}(\omega) \hat{\boldsymbol{q}}=0$, where $\hat{\boldsymbol{q}}=(\hat{p}, \hat{u})$. The eigenvalues $\omega$ are computed by setting the determinant of $\mathcal{L}$ to 0 . Once the eigenvalues are known, the eigenvectors $\hat{\boldsymbol{q}}_{j}$ and subsequently the mode-shapes $(\hat{p}, \hat{u})$ can be computed. The matrices for the Rijke tube and turbulent combustor are shown in Appendix A.

\subsection{Helmholtz solver}

Linearization of the governing equations (28) can also used to obtain the wave equation, which when transformed into frequency space and accounting for an unsteady heat release yields the thermoacoustic Helmholtz equation:

$$
\nabla \cdot\left(\bar{c}^{2} \nabla \hat{p}\right)+\omega^{2} \hat{p}=-\mathrm{i} \omega(\gamma-1) \hat{q}(\boldsymbol{x})
$$

Here, $\hat{p}$ and $\hat{q}$ denote the Fourier-transforms of the fluctuations of the pressure distribution and the local heat release rate, respectively. Note, that a $(\cdot)(t) \mapsto(\hat{\cdot})(\omega) \exp (\mathrm{i} \omega t)$ convention is used to define the Fourier-transform. $\bar{c}$ refers to the mean of the local speed of sound.

Equivalently to the previous section the heat release may be modeled in terms of an $n-\tau$-model

$$
\hat{q}=\frac{\bar{q}}{\bar{u}} n \hat{u}_{\mathrm{ref}} \exp (-\mathrm{i} \omega \tau)
$$

In contrast to network models, the Helmholtz equation accounts for the local distribution of its primitive variables and thus the interaction index $n$ and the time delay $\tau$ may be chosen to be fields of the spatial coordinate $\vec{x}$ allowing for a more flexible flame modeling. To close the problem the velocity fluctuation is linked to the pressure fluctuation by a linearized momentum balance. This yields $\hat{u}_{\text {ref }}=-\nabla \hat{p}_{\text {ref }} /(\mathrm{i} \omega \bar{\rho})$, hence we have

$$
\nabla \cdot\left(\bar{c}^{2} \nabla \hat{p}\right)+\omega^{2} \hat{p}-(\gamma-1) \frac{\bar{q}}{\bar{\rho} \bar{u}} \nabla \hat{p}_{\text {ref }} n \exp (-\mathrm{i} \omega \tau)=0
$$


Finally, a set of impedance-boundary conditions

$$
\nabla \hat{p}+\mathrm{i} \frac{\omega}{\bar{c} Z(\omega)} \hat{p}=0
$$

completes the problem definition. Here, $Z$ is the frequency-dependent boundary impedance. It can be related to the reflection coefficient $R$ used to define the boundary conditions for the network model by

$$
Z(\omega)=\frac{1-R(\omega)}{1+R(\omega)}
$$

Altogether, the thermoacoustic Helmholtz equation and the boundary conditions form the eigenvalue problem under consideration. To solve it a finite element method may be applied, as it is done with the python-based Helmholtz solver PyHoltz ${ }^{1}$ used at Technische Universität Berlin to compute results for the present study. Such a FEM discretization yields the following system of equations

$$
\underbrace{\left(\boldsymbol{K}+\omega \boldsymbol{B}+\omega^{2} \boldsymbol{M}+n \exp (-\mathrm{i} \omega \tau) \boldsymbol{Q}\right)}_{\mathcal{L}_{\mathrm{FEM}}(\omega)} \boldsymbol{p}=\mathbf{0}
$$

where $\boldsymbol{p}$ denotes the FEM discretization of the pressure fluctuation, $\boldsymbol{K}$ represents the stiffness matrix discretizing the $\nabla \cdot \bar{c}^{2} \nabla$ operation, $\boldsymbol{B}$ the boundary mass matrix modeling the boundary conditions, $\boldsymbol{M}$ the mass matrix originating from the identity operation, and $\boldsymbol{Q}$ the FEM equivalent of the heat release operation. The system can be solved by the fixed point iteration proposed by Nicoud et al. [8].

\section{Application to a Rijke Tube Model}

As a first test case a simple Rijke tube of $l=.5 \mathrm{~m}$ length is considered. The flame and reference point are located at the middle of the tube. The upstream end is assumed to be sound hard, while the downstream end is sound soft. The cold and hot gas temperature are $T_{u}=300 \mathrm{~K}$ and $T_{b}=1200 \mathrm{~K}$, respectively. The mean value for the interaction index is set to $n_{0}=1$ and the mean value for time delay to $\tau_{0}=5.34 \mathrm{~ms}$. The eigenfrequency of the first axial mode of this configuration is $\frac{\omega}{2 \pi}=187.05 \mathrm{~Hz}-0.36 \mathrm{i} \mathrm{s}^{-1}$ when computed with the network model and $\frac{\omega}{2 \pi}=187.11 \mathrm{~Hz}-0.26 \mathrm{i} \mathrm{s}^{-1}$ according $^{2}$ to the Helmholtz solution. The Helmholtz simulation used a mesh with 3336 degrees of freedom. The mode shape obtained from the simulation is depicted in figure 2 .

Because of the low growth rate, this mode can be assumed to be marginally stable. Table 1 lists the risk factors computed with the different methods and models when a standard deviation of $10 \%$ is assumed for the interaction index and the time delay.

All methods predict the risk factor to be about $52 \%$. The difference in the predictions is so small that it is negligible when considering the general modeling accuracy. In this study the evaluation of 10000 samples took minutes for the network calculations and hours for the Helmholtz computations, while the adjoint methods were performed in seconds.

Figure 3 shows how 121 uniformly distributed samples are mapped to the complex plane, for both the network and the Helmholtz model. Obviously, there is no significant difference in the results of the two methods. Moreover, even from such a few number of samples it can already be inferred that the risk factor is about $50 \%$. This shows that in contrast to MC sampling a low number of samples is required when uniform sampling is utilized.

Figure 4 further emphasizes the faster convergence of uniform sampling. It compares the risk factor estimated by uniform and MC sampling obtained from the network model with up to 1024 sample points in parameter space. While estimate found by the uniform sampling converges relatively fast and monotonically to the correct value, the estimate obtained from MC sampling is fluctuating before it stabilizes at higher sample numbers. Because the MC samples are chosen randomly, this slower convergence is a manifestation of the law of large numbers. This clearly demonstrates that a deterministic evaluation of the area at risk in parameter space is a better alternative to random-based MC sampling.

Because the adjoint methods evaluate this area, much faster than any direct method. They should be considered first when the risk factor is to be calculated. Figure 5 shows an approximation of the stability margin in parameter space computed by the second order adjoint method. Consequently, the ratio of the areas of the stable and unstable and thus the risk factor can be computed.

The Rijke tube test case demonstrated advantages of deterministic risk factor computations in the parameter space. Especially the adjoint methods have proven to be fast alternatives to MC sampling. It also validated the PyHoltz solver. The next section considers the uncertainties of a laboratory scale combustor. 


\section{Results for a laboratory-scale combustor}

The turbulent swirled combustor under investigation, designed and built at EM2C laboratory, was studied in [25,26,27]. This axisymmetric combustor (Fig. 6) consists of a cylindrical plenum of variable length $l_{1}$, a convergent duct in which a swirler induces a flow with swirl number of 0.55 , and a cylindrical combustion chamber of variable length $l_{3}$. Here, the lengths are fixed to $l_{1}=0.096 \mathrm{~m}$ and $l_{3}=0.2 \mathrm{~m}$. A mixture of methane and air at an equivalence ratio of 0.8 is injected upstream of the plenum. The operating condition 'B' of Palies et al. [26] is considered in this study. The corresponding flame has a total power of $\bar{Q}=3.03 \mathrm{~kW}$ with a mean flow velocity at the reference position of $\bar{u}_{\text {ref }}=4.16 \mathrm{~ms}^{-1}$. In [27] this geometry was labeled as configuration $\mathrm{C} 03$ and numerical and analytic computations have verified that it is marginally stable. For this computations based on comparison to harmonic-oscillator dynamics it was assumed that the system exhibits an overall acoustic damping of $\alpha=125 \mathrm{~s}^{-1}$.

This section considers measurement uncertainties for the gain $G$ and phase $\phi$ of a flame transfer function. The respective uncertainties are $\Delta G$ and $\Delta \phi$. The flame transfer function used in the computations is therefore dependent on these two additional input parameters:

$$
\operatorname{FTF}(\omega, \Delta G, \Delta \phi)=\underbrace{G(\omega) \exp (-\mathrm{i} \phi(\omega))}_{\mathrm{FTF}_{\text {measured }}(\omega)}(1+\Delta G) \exp (-\mathrm{i} \Delta \phi)
$$

Note, that this error model defines the $\Delta G$ as a relative measurement error while $\Delta \phi$ is considered an absolute measurement error. As flame response data the measured flame describing function for flame B in [27] for an amplitude level of 0.04 has been taken. The authors stated that for this low amplitude the flame response is still in the linear regime. For the adjoint perturbation to work, the flame transfer function must be differentiable with respect to its parameters. Therefore, the data has been fit to a rational function with numerator and denominator degree 10 following the method proposed in [29, 30].

Again a wave-based network model and the PyHoltz solver have been used to study the risk factor. The definition of the wave-based model is the same as in [27]. The only difference is the incorporation of the acoustic damping $\alpha$ into the model. In [27] it is neglected while solving the eigenvalue problem and substracted from the found eigenfrequency afterwards, here it is substracted from the eigenfrequency in all terms of the eigenproblem except for the flame response:

$$
\mathcal{L}(\omega)=\mathcal{W}(\omega-\mathrm{i} \alpha)+\operatorname{FTF}(\omega) Q(\omega-\mathrm{i} \alpha)
$$

In the above equation, the operator $\mathcal{W}$ comprises all terms modeling the purely acoustic wave propagation and $Q$ is the flame response operator. Hence, the growth rate of the passive solution, i.e., the solution without flame response is exactly lowered by $\alpha$. The damping is incorporated into the Helmholtz model in the same way.

This modeling of the damping has been chosen because the FTF cannot be evaluated for the real part of the eigenfrequency, as it is often done. Again, this is to retain differentiability. Because, the mode this study focuses on is marginally stable when the damping is considered, it imaginary part is negligible. Consequently, the results from this computation should not differ much from those found in [27]. Indeed, the mode found with the network model has an eigenfrequency, of $\frac{\omega}{2 \pi}=152.88 \mathrm{~Hz}-1.43 \mathrm{i} \mathrm{s}^{-1}$ which agrees well with the solution in [27], which is about $150 \mathrm{~Hz}$. The mode computed with PyHoltz feautures an eigenfrequency of $\frac{\omega}{2 \pi}=161.24 \mathrm{~Hz}-1.16 \mathrm{i} \mathrm{s}^{-1}$. The mesh for the FEM solution featured 2368 degrees of freedom. The obtained mode shape is shown in figure 8 .

The deviation in the real part of the solutions obtained with the different modeling approaches may be attributed, to the unknown shape of the heat release zone in [27]. However, the mode is close to stability and therefore a reasonable candidate for risk factor analysis.

To calculate the risk factor a relative error for the gain of $\Delta G= \pm 0.1$ and an absolute error for the phase of $\Delta \phi=0.1 \cdot 2 \pi$ have been assumed. The risk factors obtained with the different methods are enlisted in table 2 . Figure 9 shows the stability margin in the considered parameter range identified from interpolations of 10000 uniform samples and the first and second order adjoints theory.

Because the first order theory approximates the stability margin as a straight line, it only gives a rough estimate. However, the conic section obtained from second order theory coincides very well with numerically evaluated stability margin. Clearly, the theory is applicable to this case too.

\section{Conclusions and Future Work}

Immediate identification of the stability margin in parameter space enables a faster computation of the risk factor associated with marginally stable thermoacoustic modes. First and second order multi-parameter adjoint perturbation theory has been introduced to estimate the stability margin. This approach allows for a computation of the risk factor based on only one 
single solution of the eigenvalue problem describing the thermoacoustic instability. Hence, the method is several thousand times faster, than the traditional Monte Carlo simulations utilized for the problem.

It was demonstrated how the theory can be incorporated into network and Helmholtz models for the assessment of thermoacoustic instabilities. Especially, the application of the theory for the computation of the risk factor of a mode found in a laboratory scale combustor has shown that second order theory gives a very good estimate. However, cases might exist where the dependence on the uncertain parameters is strongly nonlinear. Higher order theory might then be considered. Future work will address the question of how to chose a sufficient order. A degenerate theory for the application to annular combustors, will also be the topic of future work.

The algebraic models obtained from adjoint perturbation theory are valuable for applications beyond risk factor analysis. As they allow for the cheap prediction of the stability margin, they clearly can aid design decisions of gas turbine manufacturers regarding multiple parameters. The use of second order multi-parameter adjoint perturbation theory for optimization will also be the topic of future work.

\section{References}

[1] Lieuwen, T. C., and Yang, V., eds., 2005. Combustion Instabilities in Gas Turbine Engines, Vol. 210 of Progress in Astronautics and Aeronautics. AIAA, Inc.

[2] Staffelbach, G., Gicquel, L. Y. M., and Poinsot, T., 2009. "Large eddy simulation of self-excited azimuthal modes in annular combustors". Proceedings of the Combustion Institute, 32(2), pp. 2909-2916.

[3] Wolf, P., Balakrishnan, R., Staffelbach, G., Gicquel, L. Y. M., and Poinsot, T., 2012. "Using LES to study reacting flows and instabilities in annular combustion chambers". Flow Turbulence and Combustion, 88, pp. 191-206.

[4] Dowling, A. P., 1997. "Nonlinear self-excited oscillations of a ducted flame”. J. Fluid Mech., 346, pp. 271-290.

[5] Evesque, S., and Polifke, W., 2002. "Low-order acoustic modelling for annular combustors: Validation and inclusion of modal coupling". ASME Paper GT-2002-30064.

[6] Schuermans, B., Bellucci, V., and Paschereit, C. O., 2003. "Thermoacoustic modeling and control of multi burner combustion systems". ASME paper 2003-GT-38688.

[7] Sattelmayer, T., and Polifke, W., 2003. "Assessment of methods for the computation of the linear stability of combustors". Combustion Science and Technology, 175, pp. 453-476.

[8] Nicoud, F., Benoit, L., Sensiau, C., and Poinsot, T., 2007. "Acoustic modes in combustors with complex impedances and multidimensional active flames". AIAA Journal, 45(2), pp. 426-441.

[9] Campa, G., and Camporeale, S. M., 2014. "Prediction of the thermoacoustic combustion instabilities in practical annular combustors". Journal of Engineering for Gas Turbines and Power, 136(9), p. 091504 (10 pages).

[10] Schulze, M., Hummel, T., Klarmann, N., Berger, F., Schuermans, B., and Sattelmayer, T., 2016. "Linearized Euler equations for the prediction of linear high-frequency stability in gas turbine combustors". Journal of Engineering for Gas Turbines and Power. available online, doi: 10.1115/1.4034453.

[11] Mensah, G. A., and Moeck, J. P., 2017. "Acoustic damper placement and tuning for annular combustors: An adjointbased optimization study”. Journal of Engineering for Gas Turbines and Power, 139(6), Jan, p. 061501 (9 pages).

[12] Magri, L., Bauerheim, M., and Juniper, M. P., 2016. "Stability analysis of thermo-acoustic nonlinear eigenproblems in annular combustors. part i. sensitivity". J. Comput. Phys.

[13] Magri, L., and Juniper, M. P., 2013. "Sensitivity analysis of a time-delayed thermo-acoustic system via an adjoint-based approach". Journal of Fluid Mechanics, 719, pp. 183-202.

[14] Magri, L., Bauerheim, M., Nicoud, F., Ihme, M., and Juniper, M. P., 2016. "Efficient uncertainty quantification of thermo-acoustic stability". Symposium Thermoacoustic Instabilities in Gas Turbines and Rocket Engines Paper GTRE041 .

[15] Silva, C. F., Runte, T., Polifke, W., and Magri, L., 2016. "Uncertainty quantification of growth rates of thermoacoustic instability by an adjoint helmholtz solver". ASME Paper GT2016-57659.

[16] Poinsot, T., 2013. Simulation methodologies and open questions for acoustic combustion instability studies. Annual Research Briefs, Center for Turbulence Research, Standford University. pp. 179-188.

[17] Ndiaye, A., Bauerheim, M., Moreau, S., and Nicoud, F., 2015. "Uncertainty quantification of thermoacoustic instabilities in a swirled stabilized combustor". ASME Paper(GT2015-44133).

[18] Bauerheim, M., Ndiaye, A., Constantine, P., Iaccarino, G., Moreau, S., and Nicoud, F., 2014. "Uncertainty quantification of thermo-acoustic instabilities in annular combustors". Center for Turbulence Research Proceedings of the Summer Program 2014.

[19] Bauerheim, M., Nicoud, F., and Poinsot, T., 2016. "Progress in analytical methods to predict and control azimuthal combustion instability modes in annular chambers". Physics of Fluids, 28(021303).

[20] Fernández, F. M., 2000. Introduction to Perturbation Theory in Quantum Mechanics, 1 ed. CRC Press, September.

[21] Luchini, P., and Bottaro, A., 2014. "Adjoint equations in stability analysis". Annual Review of Fluid Mechanics, 46, pp. 493-517. 
[22] Chantrasmi, T., and Iaccarino, G., 1957. "Forward and backward uncertainty propagation for discontinuous system response using the pade-legendre method". International Journal of Uncertainty Quantification, 2, pp. 125-143.

[23] Jaynes, E., 1957. "Information theory and statistical mechanics". Physical Review, 106, pp. 620-630.

[24] Crocco, L., and Mitchell, C., 1969. "Nonlinear Periodic Oscillations in Rocket Motors with Distributed Combustion". Combustion Science and Technology, 1(2), p. 146-169.

[25] Palies, P., Durox, D., Schuller, T., and Candel, S., 2010. "The combined dynamics of swirler and turbulent premixed swirling flames". Combustion and Flame, 157(9), pp. 1698-1717.

[26] Palies, P., Durox, D., Schuller, T., and Candel, S., 2011. "Nonlinear combustion instability analysis based on the flame describing function applied to turbulent premixed swirling flames". Combustion and Flame, 158(10), pp. 1980-1991.

[27] Silva, C. F., Nicoud, F., Schuller, T., Durox, D., and Candel, S., 2013. "Combining a helmholtz solver with the flame describing function to assess combustion instability in a premixed swirled combustor". Combust. Flame, 160(9), pp. 1743-1754.

[28] Silva, C., Magri, L., Runte, T., and Polifke, W., 2017. "Uncertainty quantification of growth rates of thermoacoustic instability by an adjoint Helmholtz solver". p. 011901 (11 pages).

[29] Gustavsen, B., and Semlyen, A., 1999. "Rational approximation of frequency domain responses by vector flltlng". IEEE Transactions on Power Delivery, 14(3), pp. 1052-1061.

[30] Gustavsen, B., 2006. "Improving the pole relocating properties of vector fitting". IEEE Transactions on Power Delivery, 21(3), pp. 1587-1592.

\section{Appendix A: Wave-approach matrices}

A one dimensional network model composed of a duct of length $L_{n}$ with a compact flame located at $x=b$ is considered (Fig. 10). For the Rijke tube

$$
\left[\begin{array}{cc}
-1-R_{1} \mathrm{e}^{-\mathrm{i} \omega \tau_{1}} & 1+R_{2} \mathrm{e}^{-\mathrm{i} \omega \tau_{2}} \\
\left(1-R_{1} \mathrm{e}^{-\mathrm{i} \omega \tau_{1}}\right)\left(1+\frac{\gamma-1}{\gamma \bar{p}_{1}} \beta \mathrm{e}^{-\mathrm{i} \omega \tau}\right) \frac{\bar{c}_{2}}{\bar{c}_{1}}\left(1-R_{2} \mathrm{e}^{-\mathrm{i} \omega \tau_{2}}\right)
\end{array}\right]\left[\begin{array}{c}
G_{1} \\
F_{2}
\end{array}\right]=\left[\begin{array}{l}
0 \\
0
\end{array}\right]
$$

where $\tau_{1}=2 b / \bar{c}_{1}$ and $\tau_{2}=2\left(L_{n}-b\right) / \bar{c}_{2}$ (Fig. 10).

For the turbulent combustor see Eq. (17) in [27].

\section{Tables}

Table 1: Comparison of risk factors in \% of the Rijke tube computed with the different methods. The methods are as follows US10k: uniform sampling with 10000 sample points, US1024: uniform sampling with 1024 sample points, MC10k: Monte Carlo simulation with 10000 uniformly distributed sample points: AM1 first order adjoint method, AM2: second order adjoint method.

\begin{tabular}{lccccc}
\hline & US10k & US1024 & MC10k & AM1 & AM2 \\
\hline Network & 52.13 & 50.88 & 52.15 & 52.19 & 52.15 \\
\hline Helmholtz & 51.48 & 51.37 & 51.50 & 51.56 & 51.51 \\
\hline
\end{tabular}

Table 2: Comparison of risk factors in $\%$ of the Palies configuration computed with the different methods.

\begin{tabular}{lccccc}
\hline & US10k & US1024 & MC10k & AM1 & AM2 \\
\hline Network & 74.53 & 74.12 & 75.01 & 80.88 & 74.94 \\
\hline Helmholtz & 76.34 & 75.59 & 76.80 & 83.78 & 76.82 \\
\hline
\end{tabular}




\section{Figures}

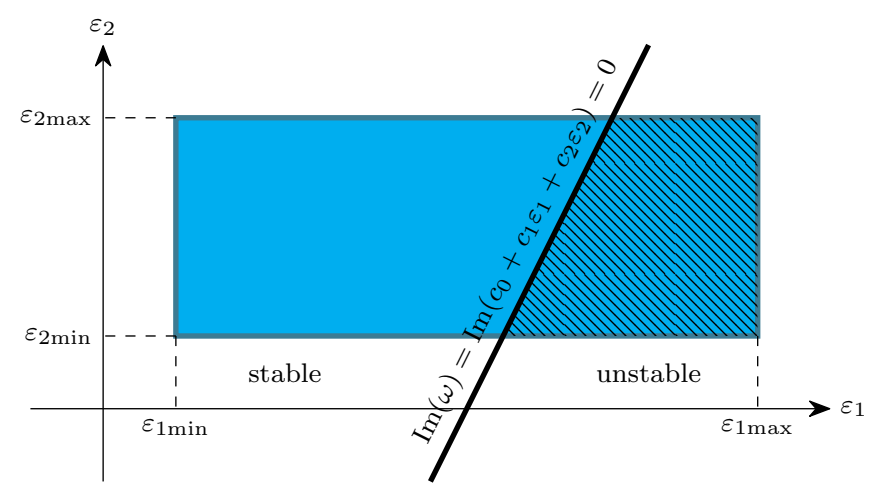

Fig. 1: Schematic illustration of risk factor evaluation in the parameter space using a linear eigenfrequency model. A uniform parameter distribution is assumed for both parameters. Thus, the parameters may take values within the blue shaded area with same probability. The linear model divides this area into two parts. The part lying in the unstable region is highlighted by the hatching. Its size is a measure for the risk factor.

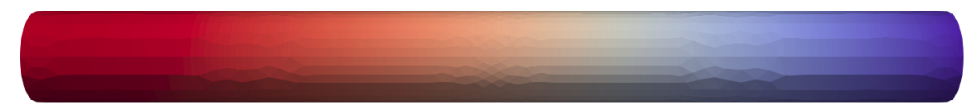

Fig. 2: The marginal stable solution under consideration. This result of the Rijke tube model was computed with PyHoltz. 

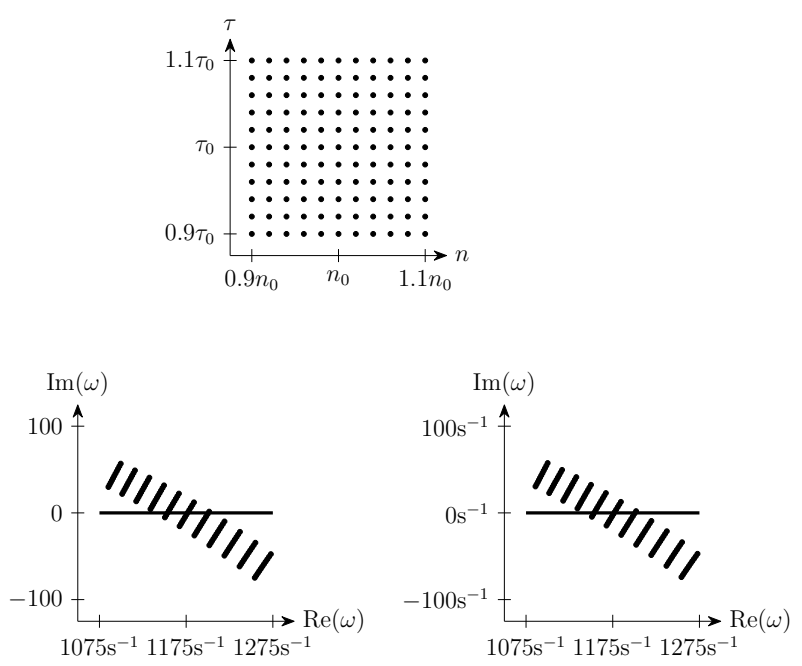

Fig. 3: Evaluation of 121 uniformly distributed samples for the Rijke tube in the parameter space top with the Helmholtz solver (bottom left) and the network model (bottom right). Obviously, there is a good agreement between the two methods. About 50 percent of the samples lie in the unstable regime, thus the risk factor is about 0.5. Though, more points were used in the present study to estimate the risk factor, the low number of samples already gives a good estimate. This would not be possible by Monte Carlo sampling.

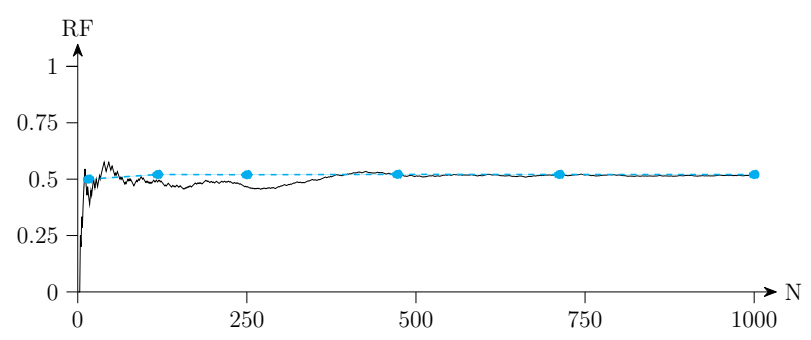

Fig. 4: Comparison of the prediction of the risk factor (RF) by use of uniformly distributed Monte Carlo samples (black) and uniformly distributed samples (cyan) for the Rijke tube network model. Obviously, uniform sampling requires fewer samples $(\mathrm{N})$ to converge than MC sampling.
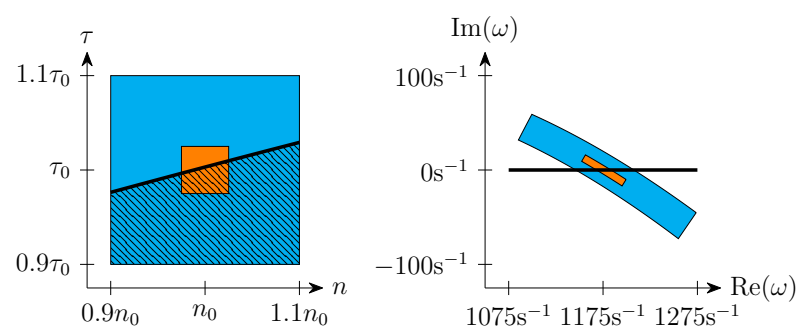

Fig. 5: Mapping of the parameters to the resulting frequencies modeled by second-order two-parameter perturbation theory. For the Rijke tube case this results in a hyperbola in parameter space separating the stable from the unstable regime. The orange shading encloses all parameter of $2.5 \%$ maximum deviation while the cyan shading corresponds to a maximum deviation of $10 \%$. 


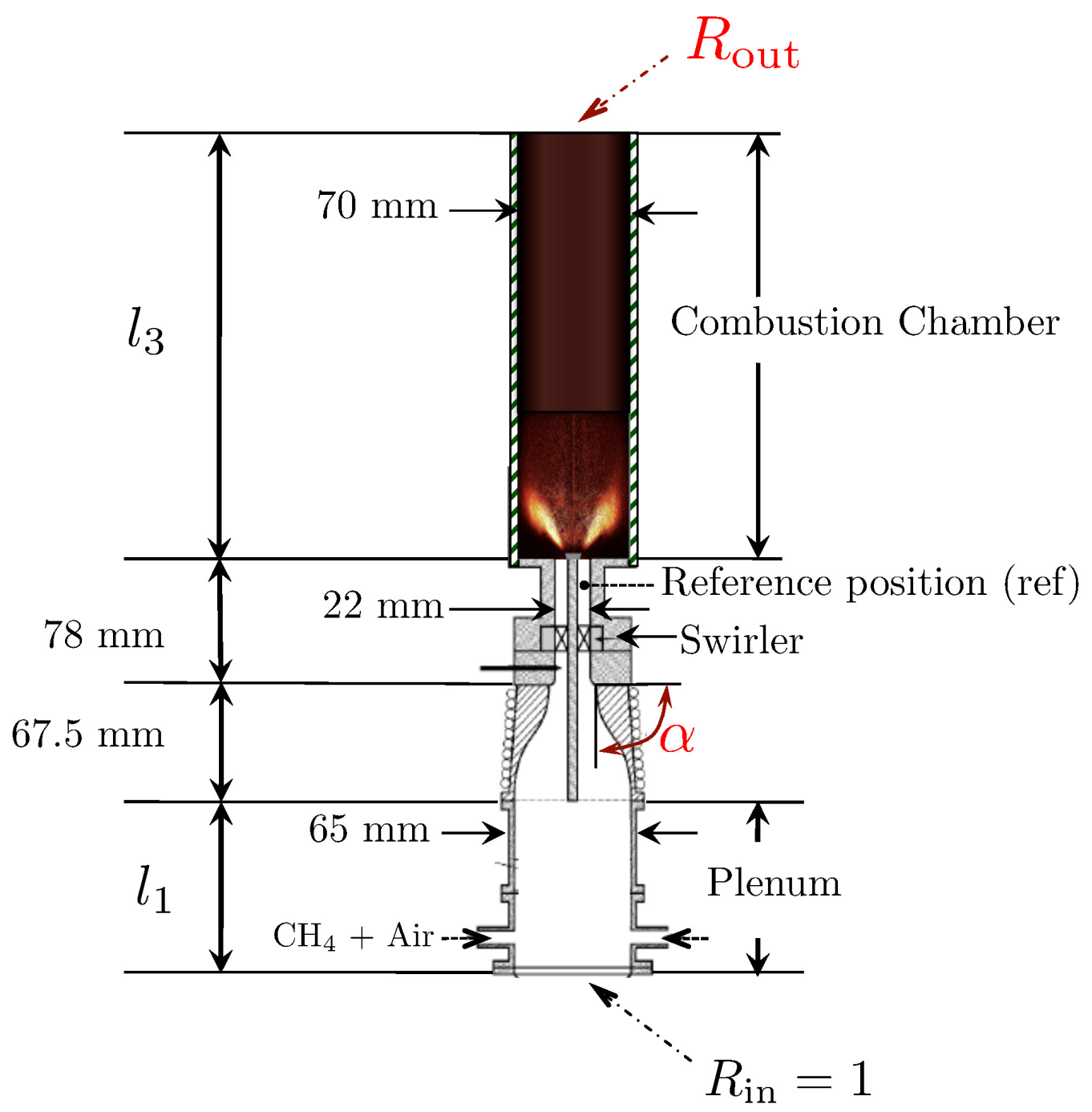

Fig. 6: Turbulent swirled combustor configuration under investigation; reproduced from Silva et al. [28]
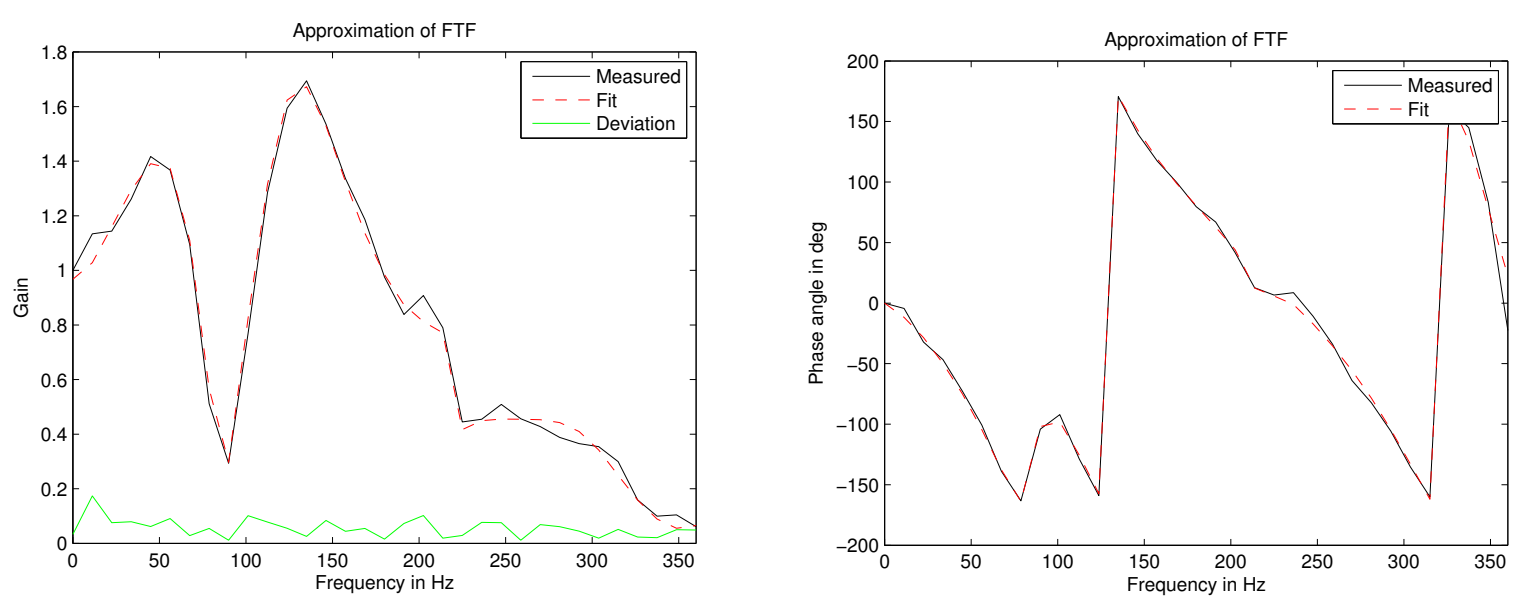

Fig. 7: Comparison of measured (black solid) and fit (red dashed) FTF. 


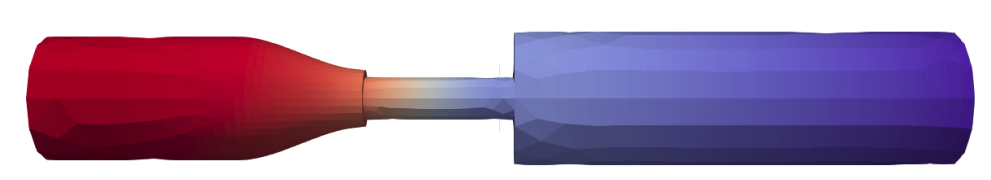

Fig. 8: Real part of the axial mode computed with PyHoltz.
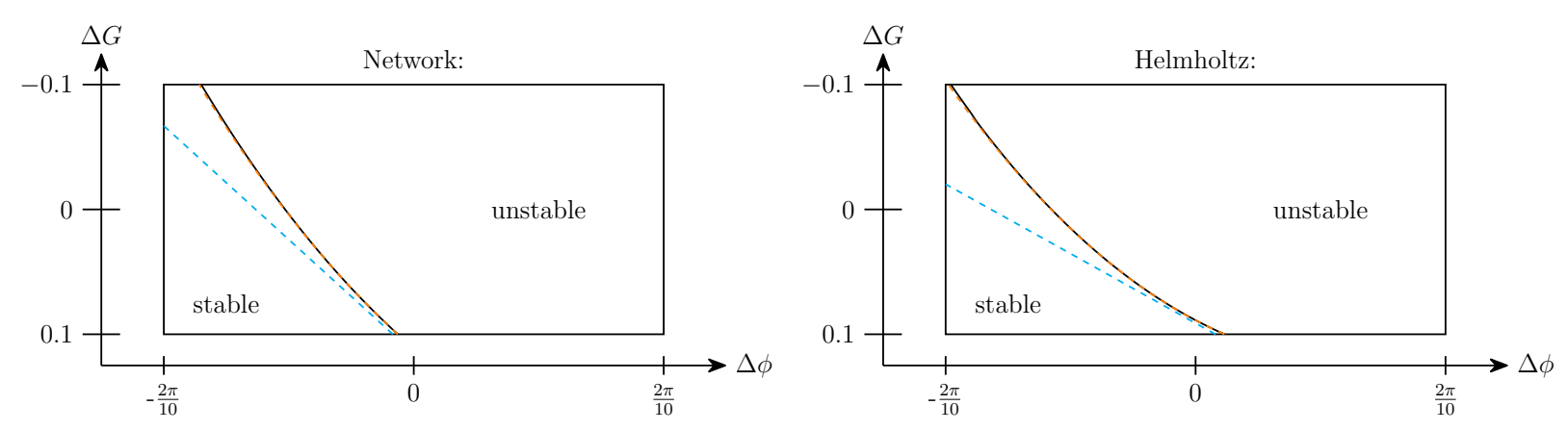

Fig. 9: Stability margin in parameter space for the two modeling approaches computed by sample interpolations (black), first (cyan) and second (orange) order adjoint theory.

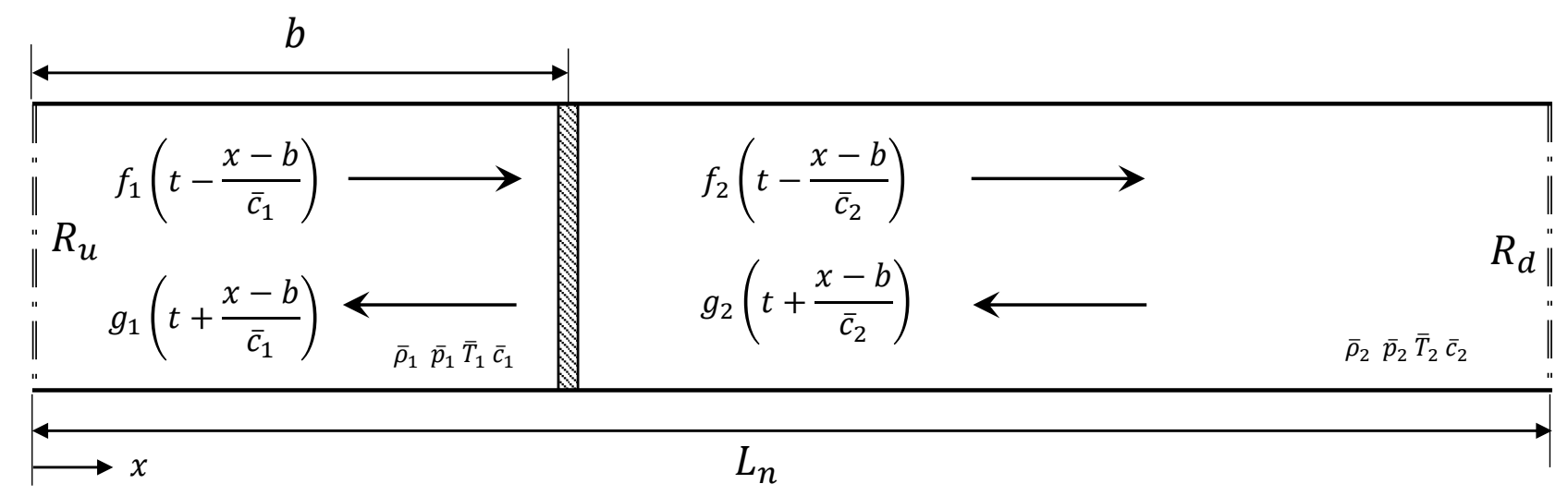

Fig. 10: WAVE-BASED THERMOACOUSTIC MODEL. THE FLAME LOCATED AT $x=b$ DIVIDES THE DUCT INTO TWO SEGMENTS. THE WAVES' REFLECTION COEFFICIENTS AT EACH END ARE $R_{1}$ and $R_{2}$. 